\title{
A pragmatical algorithm to compute the convex envelope of bathymetric surveys at variable resolutions
}

\author{
Julian Le Deunf ${ }^{a}, b, *$, Thierry Schmitt ${ }^{a}$, Yann Keramoal ${ }^{a}$ \\ a Shom (Service hydrographique et océanographique de la marine), Brest, France, julian.le.deunf@shom.fr, \\ thierry.schmitt@shom.fr,yann.keramoal@shom.fr \\ ${ }^{b}$ IMT Atlantique, Lab-STICC, UMR CNRS 6285, F-29238 Brest, France \\ * Corresponding author
}

Keywords: Quadtree indexation, Convex envelope, Bathymetric data fusion

\begin{abstract}
:
Since 1720, Shom, the French Hydrographic Service, collects information on the physical marine environment, notably bathymetric (depth) measurements. Those are at the basis of nautical products, including nautical charts, ensuring safety of navigation to the mariners. Each of Shom's bathymetric surveys are archived without order, in the dedicated Shom Bathymetric Database (SBDB), with currently over 11 400 surveys. Currently, the Tethys Shom's project is aiming at constituting the Shom bathymetric reference surface as the best bathymetric knowledge (as detailed in Le Deunf \& all, 2021, Ocean2021) based on the data in the SBDB and through which only the bathymetric data of the best quality (locally) are remaining.

In order to build this reference surface and to achieve the best possible data deconfliction and compiling in the hydrographic sense regarding to safety of navigation -, it is particularly important to compute the convex envelope of the bathymetric data sets (point cloud) composing a survey, as this multipolygon is used as a cookie-cut in the automatic process of selection of the best soundings according to the coverage and describing metadata of the parent survey. In a simplified way, the most recent and high quality survey cancels and replaces the older. The envelope of each dataset, called SME - for minimal enclosing surface must be built based on the following requirements:
\end{abstract}

- Unicity: each sounding of the dataset is included in a single SME ;

- Density: soundings which have a distance with the nearest neighbours less than 5 times of the resolution of the dataset are aggregated. If not, a new polygon is created, and eventually, the sounding is considered as an isolated sounding if it's impossible to aggregate it with neighbours;

- Representativeness: the contour (internal and external) of the mulipolygons is dilated by a buffer distance depending on the characteristics of the survey: horizontal uncertainty and resolution. That is a sensitive point to avoid removing a shoal at the border of the survey with a too loose SME while the area has not been strictly covered.

Importantly, while this polygon simplifies the geometry of the coverage, it is important that none of the soundings is filtered out through this process, as its measure might need to be displayed on the chart and might be critical for navigation. Figure 1 presents the overall workflow used to compute the multipolygon representing the convex envelope of a bathymetric point cloud. The algorithm takes as input a point cloud (xyz) file and the horizontal uncertainty associated with the soundings (originating from the use of the metadata associated to the survey). Our approach works on both geographic and projected coordinates. 


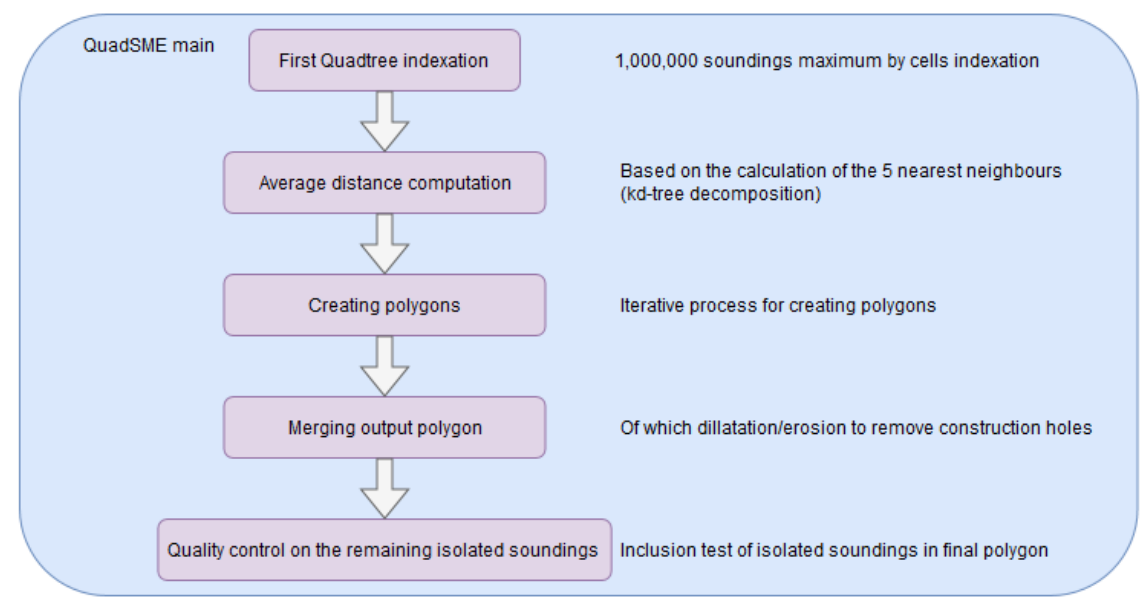

Figure 1. Overall workflow of our approach.

Figures 2 shows the results obtained on a real dataset and in particular the integration of the complex geometry and holes that may exist in this bathymetric data coverage (figure 3). Preserving holes is essential when dealing with the selection of soundings originating from overlapping surveys, in order to prevent from an excessive filtering of valuable information.
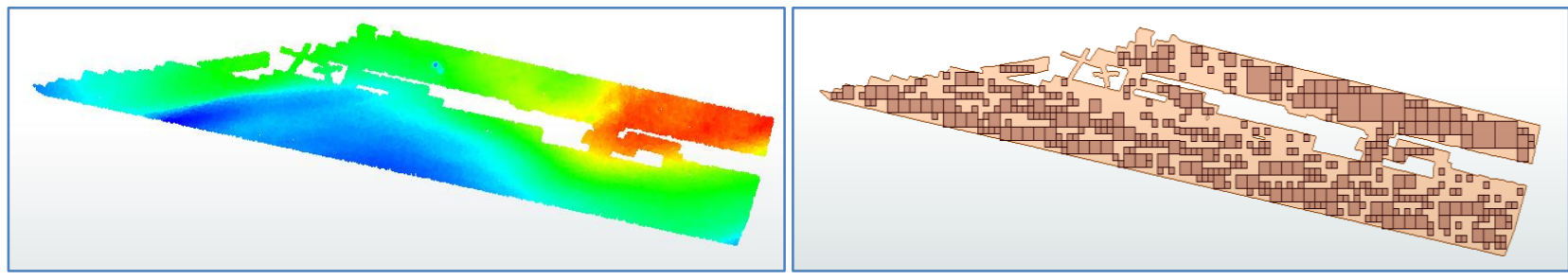

Figure 2. On left a bathymetric survey (point cloud), on right its corresponding convex envelope with holes preserved. Also note the corresponding quadtree decomposition.

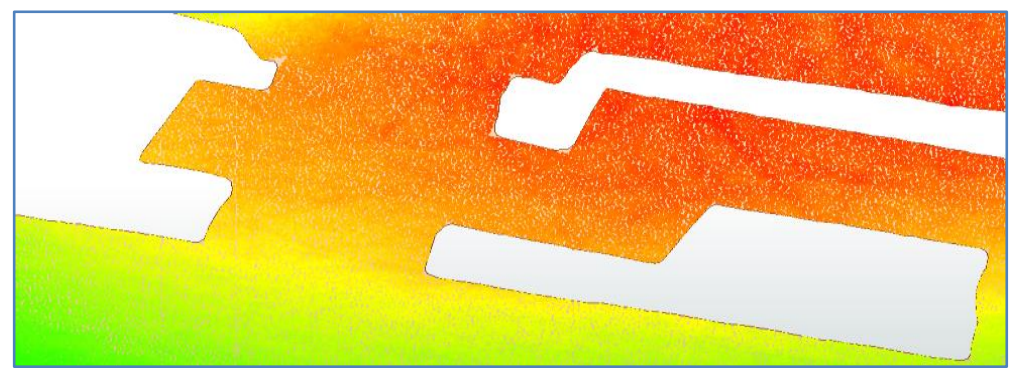

Figure 3. Zoom on particular region of the figure 2 bathymetric survey.

This algorithm has been developed using Python and could be the subject of a Git repository after its release. The full paper will present in detail the global workflow and in particular the iterative process in the Quadtree SME block (see figure 1). It will also show the differences with classical approaches based on convex hull algorithms (as Graham \& all, 1983), but also the associated processing times on large datasets (order of magnitude of several million soundings). While the amount of bathymetric data is increasing with the improvement of sensor resolution and the widespread use of new technologies as lidar, this algorithm provides to hydrographers a new efficient tool to automate a previously tedious manual task and to standardize practices by reducing the subjectivity of a manual digitalization. 